\title{
Polyfluorene as a model system for space-charge-limited conduction
}

\author{
M. Arif \\ M. Yun \\ S Gangopadhyay \\ K. Ghosh \\ Missouri State University \\ L. Fadiga \\ Missouri State University
}

See next page for additional authors

Follow this and additional works at: https://bearworks.missouristate.edu/articles-cnas

\section{Recommended Citation}

Arif, Mohammad, Minseong Yun, Shubhra Gangopadhyay, K. Ghosh, L. Fadiga, F. Galbrecht, Ullrich Scherf, and Suchi Guha. "Polyfluorene as a model system for space-charge-limited conduction." Physical Review B 75, no. 19 (2007).

This article or document was made available through BearWorks, the institutional repository of Missouri State University. The work contained in it may be protected by copyright and require permission of the copyright holder for reuse or redistribution.

For more information, please contact BearWorks@library.missouristate.edu. 
Authors

M. Arif, M. Yun, S Gangopadhyay, K. Ghosh, L. Fadiga, F. Galbrecht, U. Scherf, and S. Guha 


\title{
Polyfluorene as a model system for space-charge-limited conduction
}

\author{
M. Arif, ${ }_{1}^{1}$ M. Yun, ${ }^{2}$ S. Gangopadhyay, ${ }^{2}$ K. Ghosh, ${ }^{3}$ L. Fadiga, ${ }^{3}$ F. Galbrecht, ${ }^{4}$ U. Scherf, ${ }^{4}$ and S. Guha ${ }^{1, *}$ \\ ${ }^{1}$ Department of Physics and Astronomy, University of Missouri, Columbia, Missouri 65211, USA \\ ${ }^{2}$ Department of Electrical Engineering, University of Missouri, Columbia, Missouri 65211, USA \\ ${ }^{3}$ Department of Physics, Missouri State University, Springfield, Missouri 65804, USA \\ ${ }^{4}$ Bergische Universität Wuppertal, Makromolekulare Chemie, Wuppertal D-42097, Germany \\ (Received 11 October 2006; revised manuscript received 8 February 2007; published 3 May 2007)
}

\begin{abstract}
Ethyl-hexyl substituted polyfluorene (PF) with its high level of molecular disorder can be described very well by one-carrier space-charge-limited conduction for a discrete set of trap levels with energy $\sim 0.5 \mathrm{eV}$ above the valence-band edge. Sweeping the bias above the trap-filling limit in the as-is polymer generates a set of exponential traps, which is clearly seen in the density of states calculations. The trapped charges in this set of traps have very long lifetimes and can be detrapped by photoexcitation. Thermal cycling the PF film to a crystalline phase prevents the creation of additional traps at higher voltages.
\end{abstract}

DOI: 10.1103/PhysRevB.75.195202

PACS number(s): 72.80.Le, 73.61.Ph, 85.60.Jb

\section{INTRODUCTION}

Polyfluorenes (PFs) have emerged as an especially attractive conjugated polymer $(\mathrm{CP})$ due to their strong blue emission and excellent electronic properties, and thus great prospects for device applications. ${ }^{1,2}$ Such applications rely on charge-carrier injection and transport. ${ }^{3}$ Recently, it was shown that the parallel electron mobilities in field-effect transistors based on PF copolymers are one of the highest: $10^{-3}-10^{-2} \mathrm{~cm}^{2} / \mathrm{V} \mathrm{s}{ }^{4}$ Charge-carrier transport in CPs mainly occurs by variable-range hopping, ${ }^{5}$ where polarons are the actual carriers, ${ }^{6}$ and is strongly affected by the presence of traps at the metal-organic interface and in the bulk. Such trap states (shallow or deep) are favorable energy states; below the conduction-band edge, these states can capture an electron, and above the valence-band edge, can capture a hole.

Impurities and structural defects typically result in discrete trap states within the energy gap. ${ }^{7}$ Thermally stimulated currents ${ }^{8}$ photoinduced absorption, ${ }^{9}$ impedance spectroscopy,${ }^{10}$ and current-voltage $(I-V)$ characteristics in the space-charge-limited current (SCLC) regime ${ }^{11}$ are some of the experimental methods for the detection of trap states. The latter yields information on the energy and density of traps as well as charge-carrier mobilities. A recent work on polyacenes shows that a percolative fluctuation process gives a better insight into the electronic conditions determining the crossover from Ohmic to SCLC regime. ${ }^{12}$

SCLC-based models have been used to study trap states in organic molecules and polymers over the last $\sim 40$ years. Trapping of carriers in multitrap levels has been observed in thiophene-based films. ${ }^{13}$ Shallow trapping with a single trap level in naphthalene and anthracene ${ }^{14}$ was clearly observed in the $I$ - $V$ characteristics, which is distinguished by four different regions. These include (a) the Ohmic region supported by thermal carrier generation, (b) Child's law in the presence of shallow trapping, (c) trap-filled limit, and (d) Child's law in the absence of trapping. The above regions rely on the idea that the energy level of any given trap has a precisely defined value. Undoubtedly, for single-crystal materials of high chemical and structural purity, this is a good approximation for current injection. On the other hand, electrons or hole traps in amorphous insulators and semiconductors do not have a uniquely defined environment because of the large structural disorder. As a result, isolated discrete energy levels for traps in these materials do not provide an adequate model.

Charge injection in blue-emitting polymers is a topic of heavy debate. In this work, we show that charge injection in ethyl-hexyl substituted PF can be described very well by one-carrier SCLC-voltage characteristics. This work provides data on charge injection in a blue-emitting polymer with a high level of molecular disorder successfully modeled by discrete energy levels for traps. These unexpected findings suggest that charge injection and transport occur through regions of ordering in the polymer. The initial $I-V$ run exhibits a shallow trap behavior for holes, after which subsequent bias sweeps show a distribution of trap energies in the as-is polymer. The density of traps $N_{t}$, their energy levels, and the mobility of carriers are obtained from the $I-V$ characteristics.

\section{EXPERIMENTAL DETAILS}

\section{A. PF structure}

Almost all PF derivatives utilize solubilizing side chain substituents anchored at the bridging carbon atom. ${ }^{15}$ Use of side chains containing chiral centers creates opportunities for optical activity and the emission of circularly polarized light. Distinct differences in the optical and electronic properties are observed in two members of the PF family: poly $\left[9,9^{\prime}\right.$-(di $n$, octyl)fluorene] (PF8) and poly[9, $9^{\prime}$-(di 2-ethylhexyl)fluorene] (PF2/6). These polymers are characterized by thermotropic mesophases including a nematicliquid crystalline $\left(n\right.$-LC) phase, typically above $150{ }^{\circ} \mathrm{C} .{ }^{16,17}$

PF8 with two linear octyl side chains is characterized by the occurrence of different solid-state phases. ${ }^{16}$ Their formation strongly depends on the conformation of side chains ${ }^{18}$ and processing conditions that further complicate the discussion of solid-state electronic properties in PF8. PF2/6 with its branched alkyl side chains has a high level of molecular disorder but forms one solid-state phase. In this hexagonal 
phase, the individual PF2/6 chains adopt a fivefold helix $(5 / 2)$ with coherence lengths exceeding $50 \mathrm{~nm} \cdot{ }^{15,17}$ Since the application of SCLC technique is based on the purity and crystallinity of a material, PF2/6 serves as a unique "test" case because of its structural properties.

\section{B. Methodology}

The indium tin oxide (ITO) layer was grown in patterned structures on glass slides using pulsed laser deposition (PLD). Poly(ethylenedioxythiophene)-poly(styrenesulfonate) (PEDOT-PSS) was first spin coated onto the ITO-coated slides (thickness $\sim 80 \mathrm{~nm}$ ), on top of which PF2/6 was spin coated from a toluene solution $(10 \mathrm{mg} / \mathrm{ml})$. This was again coated with an $80 \mathrm{~nm}$ PEDOT-PSS layer. The top Al electrode was deposited by thermal evaporation at a base pressure of $10^{-6}$ mbar. These diode structures were then encapsulated. All fabrication steps were carried out in a nitrogen glovebox. Light-emitting-diode structures were also fabricated by directly capping the PF2/6 layer either with $\mathrm{Al}$ or with $\mathrm{Ca}$ (capped with Al). The thickness of the PF2/6 layer was $100 \mathrm{~nm}$, device area was $(2-4) \times 10^{-4} \mathrm{~cm}^{2}$, and each sample held 25 devices. ITO/PEDOT-PSS/(PF2/6)/PEDOT$\mathrm{PSS} / \mathrm{Al}$, ITO/PEDOT-PSS/(PF2/6)/Al, and ITO/PEDOT$\mathrm{PSS} /(\mathrm{PF} 2 / 6) / \mathrm{Ca}-\mathrm{Al}$ structures are referred to as sample A, sample B, and sample C, respectively. Sample A is truly a hole-only device.

We prepared another device (sample D) where the PF2/6 film was annealed to induce the hexagonal crystalline phase using similar temperature steps used by Tanto et al. in bulk PF2/6. ${ }^{15}$ After spin coating the PF2/6 layer on PEDOT-PSS coated ITO, the sample was placed in an oven (inside the glovebox) at $80{ }^{\circ} \mathrm{C}$ for $15-20 \mathrm{~h}$. Then the temperature was slowly raised to $150{ }^{\circ} \mathrm{C}$ at the rate of $10^{\circ} \mathrm{C} / \mathrm{h}$ and left for $2 \mathrm{~h}$ at the highest temperature. It was then cooled to room temperature at $1{ }^{\circ} \mathrm{C} / \mathrm{min}$. The device structure was similar to sample A, with a top PEDOT-PSS layer capped with Al.

The dielectric constant of the polymer (both as-is and annealed) was measured from $\mathrm{Al} /(\mathrm{PF} 2 / 6) / \mathrm{Al}$ structures by capacitance methods. For both the as-is and annealed films the dielectric constant was measured as $\sim 2.7$ at $10 \mathrm{kHz}$, with a rms voltage of $2.1 \mathrm{~V}$. The $I-V$ measurements were carried out by a Keithley 236 source meter using manual probes. For the low-temperature measurements, a CTI closed-cycle refrigerator was used.

\section{CURRENT-VOLTAGE CHARACTERISTICS}

\section{A. As-is PF2/6 film}

Figure 1 shows the current density $(J)$ vs voltage $(V)$ of sample A for many bias sweeps. The very first measurement clearly shows all four regions, characteristic of a one-carrier single set of traps. These include the (1) Ohmic region, where the current density is proportional to the voltage, $J=q n_{0} \mu V / d, n_{0}$ being the free carrier density, $d$ the thickness of the polymer layer, and $\mu$ the carrier mobility; (2) SCLC trap-limited region, where the current density is given by $J=9 \epsilon \epsilon_{0} \mu \Theta V^{2} / 8 d^{3}$, where $\Theta$ is the trapping fraction, $\epsilon$ is the dielectric constant of the polymer, and $\epsilon_{0}$ is the permittivity

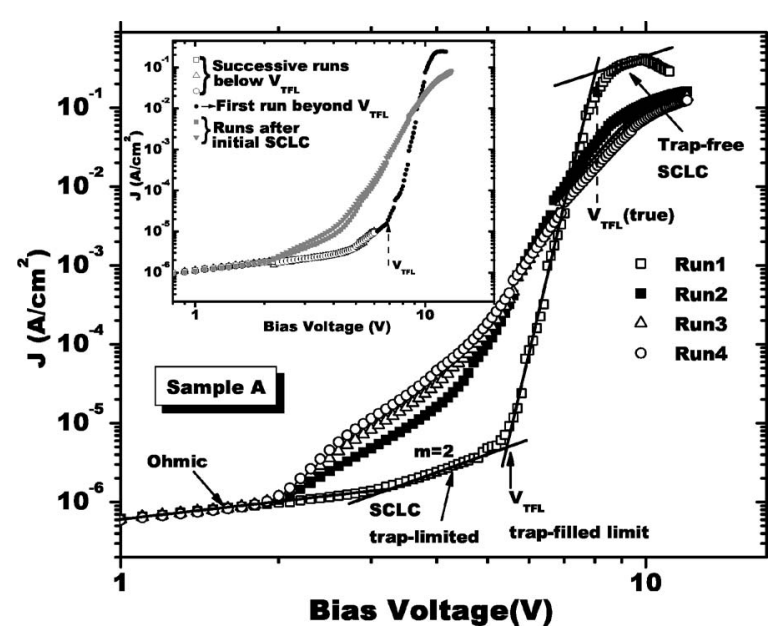

FIG. 1. $J-V$ characteristics for sample A. The initial sweep (Run 1) shows all four distinct regions, characteristic of a shallow-trap SCLC behavior with slope $(m)=2$ preceding $V_{T F L}$. Subsequent runs show higher values of $m$. The inset shows $J$ vs $V$ from another device of the same sample. The open symbols denote three successive $J$ - $V$ sweeps below $V_{T F L}$; $\bullet$ shows the very first run upto trapfree SCLC voltages. Successive sweeps are shown by the solid symbols.

of free space; (3) trap-filled limit, where the onset trap-filled voltage $V_{T F L}$ results in the density of traps $\left(N_{t}\right)$, which is given by

$$
N_{t}=\frac{3}{2} \frac{\epsilon \epsilon_{0} V_{T F L}}{q d^{2}}
$$

and (4) the final region is the current only limited by the space charge and free from the influence of traps. This trapfree SCLC region is similar to region (2) with $\Theta=1$. The trapping parameter in the trap-limited conduction region is given by

$$
\Theta=\frac{N_{v}}{N_{t}} \exp \left(-\frac{E_{t}}{k T}\right)
$$

where we assume that the top of the valence-band edge is $E_{v}=0 ; N_{v}$ is the density of states within $k T$ of the valenceband edge. Since sample A is a hole-only device, the traplimited current arises from shallow hole traps. This is similar to what is seen in pure organic crystals such as naphthalene. ${ }^{14}$ One can extract all the relevant parameters from the four regions. Region 4 fits very well with $J \propto V^{2}$; from the slope, we extract the value of $\mu$. (Here we assume field-independent mobilities.) This is then substituted in the trap-limited region (2) to obtain the value of $\Theta$, and by using Eq. (2), we evaluate $E_{t}$. We use $N_{v}=3 \times 10^{21} \mathrm{~cm}^{-3} \cdot{ }^{12}$ Since the transition region from $V_{T F L}$ to trap-free SCLC is not perfectly vertical (due to reasons discussed later), we use $V_{T F L}$ (true), which is the intersection of trap-free SCLC and the vertical rise, in Eq. (1).

The $I-V$ curves of samples $\mathrm{B}$ and $\mathrm{C}$ are not shown here but are similar to sample A. Although Ca provides a very low barrier for electrons, the device is predominantly hole-like. This is most probably because the PLD grown ITO with 
TABLE I. Hole mobility, trap density, and energy of the traps obtained from shallow-trap $J$ - $V$ characteristics.

\begin{tabular}{lccc}
\hline \hline Sample & $\mu\left(\mathrm{cm}^{2} / \mathrm{V} \mathrm{s}\right)$ & $N_{t}\left(\mathrm{~cm}^{-3}\right)$ & $E_{t}(\mathrm{eV})$ \\
\hline A & $1.8 \times 10^{-5}$ & $1.8 \times 10^{17}$ & $0.52 \pm 0.03$ \\
B & $2.5 \times 10^{-7}$ & $2.1 \times 10^{17}$ & $0.35 \pm 0.03$ \\
C & $1.9 \times 10^{-6}$ & $1.5 \times 10^{17}$ & $0.47 \pm 0.04$ \\
\hline \hline
\end{tabular}

PEDOT-PSS contact is a better injector of holes than the metallic ones are for electrons. Single-trap SCLC behavior is observed in the very first run for all sets of devices, and subsequent measurements show an exponential trap behavior. Table I lists the mobility, trap density, and energy of the traps obtained from the shallow-trap $J-V$ characteristics for all three samples. The hole mobilities are in the range of $10^{-5}-10^{-7} \mathrm{~cm}^{2} / \mathrm{V}$ s. The trap densities are of the order of $10^{17} \mathrm{~cm}^{-3}$ and the average trap energy of PF2/6 from all three samples is $\sim 0.5 \mathrm{eV}$.

Our recent work on capacitance-voltage characteristics from PF2/6-based metal-insulator-semiconductor (MIS) diode, where as-is PF2/6 layer was the active semiconductor layer and $\mathrm{Al}_{2} \mathrm{O}_{3}$ was the dielectric layer, yields the concentration of localized charges as $5.7 \times 10^{17} \mathrm{~cm}^{-3},{ }^{19}$ similar to values of $N_{t}$ deduced here. The mobilities extracted in this work from the SCLC model are in good agreement with other works; time of flight and dark injection space-chargelimited current transient measurement from a PF copolymer yields hole mobilities in the range of $10^{-5}-10^{-6} \mathrm{~cm}^{2} / \mathrm{V} \mathrm{s} .^{20}$ Thermally stimulated current measurements from doped PF2/6 yield $0.24 \mathrm{eV}$ as the effective hole trap depth; lower trap energies are expected for a doped polymer compared to an undoped one, as is the case in this work. ${ }^{21}$

For all devices after the initial run, the $J-V$ characteristics resemble that of an exponential set of traps distributed continuously in energy (where $J \propto V^{m}$ ), as shown in Fig. 1. The slope of the trap-limited region increases until $m=6$, after which the $J-V$ characteristics do not change for subsequent measurements. These additional traps are created by the injected carriers at high fields. We verified this by sweeping the voltage of a different device (in sample A) just below the trap-filled voltage and repeated the measurements several times; the $J-V$ characteristics do not change at all, as shown by the open symbol in the inset of Fig. 1, as long as the voltage is below $V_{T F L}$. Again, after running the device once until trap-free SCLC, subsequent runs show the characteristics of a distribution of traps with $m>2$. It is due to this generation of additional traps that the transition region between $V_{T F L}$ and trap-free SCLC deviates from being perfectly vertical even in the first bias sweep. Moreover, the decrease in current at higher fields (trap-free SCLC region) suggests both emptying of traps ${ }^{14}$ and additional generation of trap states.

The inset of Fig. 2 shows the effect of detrapping by photoexcitation. The $457 \mathrm{~nm}$ line of an $\mathrm{Ar}^{+}$laser was used as the excitation source. In the figure, runs 5-7 indicate $J-V$ characteristics before photoexcitation. The sample was illuminated for $\sim 15 \mathrm{~min}$; remeasuring the current for a forward bias sweep shows the Ohmic and SCLC regions quite clearly



FIG. 2. DOS distribution as a function of the quasi-Fermi level for the initial, trap-filled, and after photoexcitation bias sweeps in sample $\mathrm{C}$. The inset shows successive $J$ - $V$ curves after running the device many times. The bottom curve in the inset was measured after photoexcitation.

(as seen in the bottom curve of the inset). Although the $J-V$ characteristics after photoexcitation reflect emptying of traps, it does not revert back to the initial shallow-trap behavior. Since the trap states are more localized compared to the transport states, transitions from a localized trap state to the continuum are typically forbidden in organic semiconductors because of the selection rules. ${ }^{7}$ The incident light generates excitons (both singlet and triplet), where the triplet excitons are also effective as detrapping agents. ${ }^{14}$ Typically free carriers are generated by autoionization, which then recombine with the trapped charges, resulting in a detrapping process. ${ }^{7}$ After photoexcitation, the Ohmic region extends for higher bias values, suggestive of the introduction of free charge carriers by the photoexcitation process. Without photoexcitation, the lifetime of trapped charges is many hours. The exact nature of these long lifetimes is not known; it is being currently investigated.

\section{Density of states calculation}

When traps are not discrete but have a distribution of energies, the slope of the $J-V$ curve is no longer 2 , and changes continuously. The density of states (DOS) distribution $\left[g\left(E_{F}\right)\right]$ can be obtained by the differential method proposed by Nespurek and Sworakowski. ${ }^{23}$ In this scheme, the DOS at the quasi-Fermi level is given by

$$
g\left(E_{F n}\right)=\left[\frac{\chi \epsilon \epsilon_{0}}{e d^{2} k T}\right]\left(\frac{V}{m(V)-1}\right),
$$

where $m(V)=d(\ln J) / d(\ln V) .{ }^{24} \chi$ is a correction factor for the nonuniformity of the internal field; here we take its value as 1. The position of the quasi-Fermi level is $E_{F n}=k T \ln \left(e N_{v} V \mu / J d\right)$. This method is sensitive to the DOS at the quasi-Fermi level.

Figure 2 shows the calculated DOS as a function of the quasi-Fermi level for three different situations in sample $\mathrm{C}$ using the method in Ref. 23: (i) the very first bias sweep, 
which resembles the first run shown in Fig. 1; (ii) after several bias sweeps when the traps are already filled (run 5, Fig. 2 inset); and (iii) after photoexcitation when the traps are emptied. Initially, the DOS is $\sim 7 \times 10^{17} \mathrm{~cm}^{-3} \mathrm{eV}^{-1}$ centered at $\sim 0.5 \mathrm{eV}$. Since the DOS calculation provides the distribution of traps with respect to the quasi-Fermi energy level, traps created at high fields after several bias sweeps show their distribution as $\sim 2 \times 10^{18} \mathrm{~cm}^{-3} \mathrm{eV}^{-1}$ at an energy of $0.35-0.4 \mathrm{eV}$. After photoexcitation, the DOS remains at $2 \times 10^{18} \mathrm{~cm}^{-3} \mathrm{eV}^{-1}$ with a shift of the quasi-Fermi level to $\sim 0.5 \mathrm{eV}$, similar to the first run, suggesting partial emptying of traps. Since the DOS values remain higher compared to the very first run even after the traps are emptied, it is a clear indication that additional traps are created at higher voltages. Generation of trap states has also been observed by Lang et al. in pentacene single crystals with bias-stress, ${ }^{22}$ however, in their work the DOS values are not calculated.

The origin of the initial set of traps could be structural or chemical defects, which typically give discrete trap states. As-is PF2/6 film shows some signature of the hexagonal ordering at room temperature. ${ }^{15}$ The defects may occur at the grain boundaries in the polycrystalline layers, resulting in shallow-trap SCLC. The exponential set of traps that are created may arise from hydrogen- and oxygen-induced defects. ${ }^{22} \mathrm{PF} 2 / 6$ is known for its fluorenone defects at the bridging carbon atom ${ }^{2}$ application of high voltages may create more of these defects.

\section{Temperature-dependent $I-V$}

Temperature-dependent $I-V$ measurements yield alternate methods to estimate $N_{t}$ and $E_{t}$ by determining $\Theta$ as a function of temperature [see Eq. (2)]. Unfortunately, since the shallow-trap behavior is seen only in the first bias sweep here, and driving the device above $V_{T F L}$ results in exponential trap levels, discrete trap SCLC model cannot be used to extract $\Theta$ as a function of temperature. However, temperature-dependent $I-V$ measurements allow us to estimate the density of traps induced at higher voltages. The temperature-dependent $I-V$ was measured after running the device to higher voltages for a few times, ensuring steady $I-V$ characteristics.

Figure 3 shows $J$ vs $V$ for sample A measured at different temperatures. Only a section of $J-V$ characteristics is shown to highlight the voltage at which these curves cross each other. At the crossover voltage $\left(V_{c}\right)$, the current is temperature independent, denoting that the activation energy is zero. This voltage is related to $N_{t}$ by $V_{c}=q N_{t} d^{2} / 2 \epsilon \epsilon_{0} .{ }^{25}$ Using $V_{c}=6.5 \mathrm{~V}$, we obtain $N_{t}$ as $2 \times 10^{17} \mathrm{~cm}^{-3}$. The inset of Fig. 3 shows that $m$ (obtained by fitting only a narrow region of the $J-V$ curve) increases as a function of inverse temperature. A similar temperature-dependent behavior is observed for the other samples as well. The linear dependence of the slope $(m)$ with inverse temperature further establishes the validity of the SCLC model for our PF2/6-based diodes.

\section{B. Thermal-cycled PF2/6 film}

Figure 4 shows the $J-V$ characteristics of sample $\mathrm{D}$, where the PF2/6 film is in a semicrystalline phase after annealing.

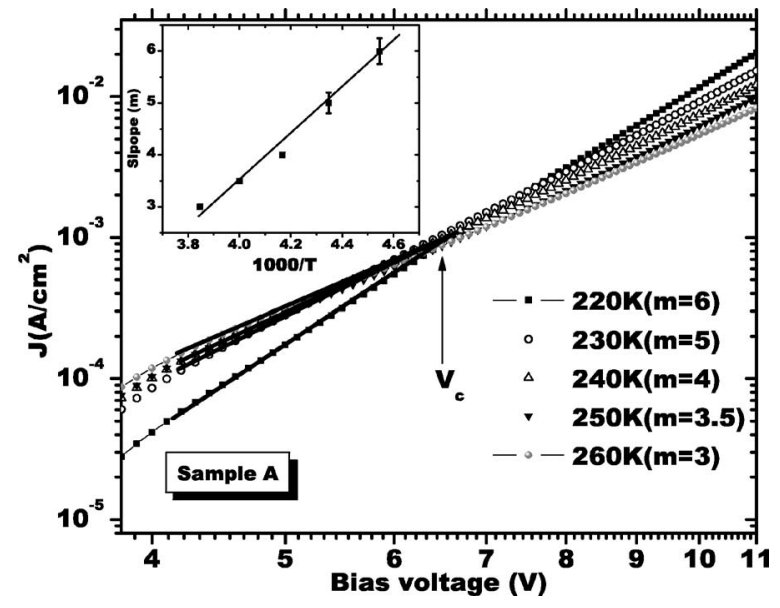

FIG. 3. $J$ vs $V$ as function of temperature for sample A. The crossover voltage is at $6.5 \mathrm{~V}$. The inset shows $m$ as a function of inverse temperature.

The exact crystallinity of these films has not been determined, but our preliminary atomic force microscopy studies from annealed PF2/6 films show average footprint area of $\sim 10^{4} \mathrm{~nm}^{2}$. Devices fabricated from this phase also show discrete traps under SCLC with all four regions. The current density in the trap-limited and trap-free SCLC regions agrees very well with a $V^{2}$ dependence. The inset shows successive $J-V$ runs from another device of the same sample; the slope of the trap-limited region no longer changes, clearly indicating that no additional traps are being created at higher voltages when the PF2/6 film is in the semicrystalline form. These measurements were repeated for several devices of sample $\mathrm{D}$, and for all of them, the $J-V$ characteristics are similar for successive runs with no additional creation of trap states.

Table II compares the mobilities, density, and energy of the trap states in as-is and annealed (sample D) PF2/6 devices. These results are from more than ten devices of each sample. The trap densities remain the same, indicating that the structural or chemical defects in semicrystalline PF2/6 is similar to the as-is polymer. The hole mobility is enhanced

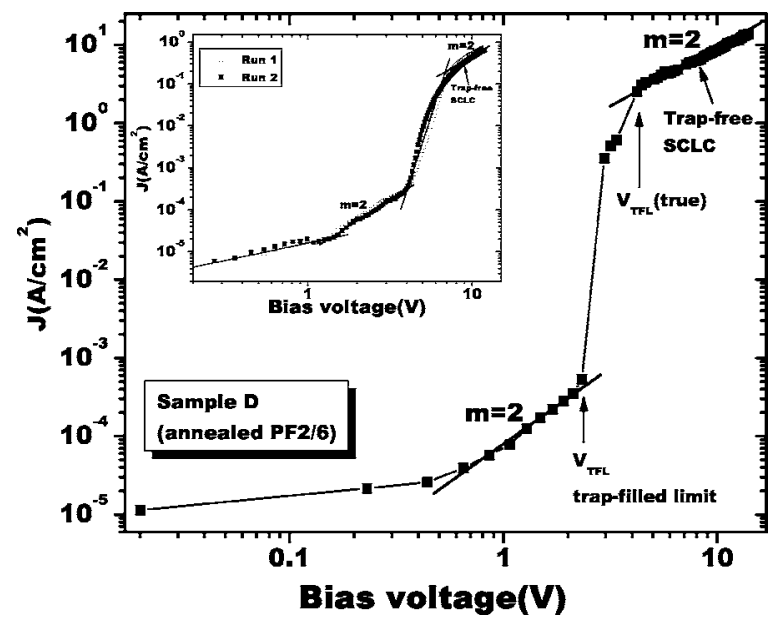

FIG. 4. $J$ vs $V$ characteristics for sample D. The inset shows two subsequent runs from another device of the same sample. 
TABLE II. Hole mobility, trap density, and energy of the traps for as-is and annealed PF2/6 devices.

\begin{tabular}{lccc}
\hline \hline Sample & $\mu\left(\mathrm{cm}^{2} / \mathrm{V} \mathrm{s}\right)$ & $N_{t}\left(\mathrm{~cm}^{-3}\right)$ & $E_{t}(\mathrm{eV})$ \\
\hline As-is & $4 \times 10^{-5}-2.6 \times 10^{-7}$ & $(1.1-1.8) \times 10^{17}$ & $0.35-0.52$ \\
Annealed & $2.3 \times 10^{-4}-2.1 \times 10^{-5}$ & $(0.7-1.5) \times 10^{17}$ & $0.38-0.45$ \\
\hline \hline
\end{tabular}

by more than an order of magnitude in the annealed sample. Such an enhancement upon annealing has been reported in PF8-based devices. ${ }^{26}$ We point out that the as-is samples represented in this table include samples B and C (without the top PEDOT-PSS layer), which show slightly lower values of $E_{t}$. Although these samples behave as one-carrier hole-only devices, there may be some electron injection, resulting in slight variations.

A striking feature of sample D is that additional traps are not created upon subsequent bias sweeps unlike as-is PF2/6. Most likely this reflects that the additional trap states arise from structural disorder due to injected carriers at voltages higher than $V_{T F L}$. In the hexagonal phase, the polymer chains adopt a specific orientation preventing such disorders.

Discrete set of traps as seen in the $J-V$ characteristics for both as-is and annealed PF2/6 indicates that current injection is primarily governed by the ordered regions in the film and does not depend on the overall bulk film properties. This is corroborated by our capacitance-voltage measurements of PF2/6-based MIS diodes, where the equivalent parallel conductance demonstrate the presence of shallow-trap states with a single time constant. ${ }^{19}$

\section{CONCLUSIONS}

In conclusion, the single-trap level SCLC model for charge injection, believed to be applicable only for crystalline materials of high chemical and structural purity, is an excellent model for a class of blue-emitting polymers. PF2/6 with its high level of molecular disorder is an exemplary system, where the SCLC model within discrete level shallow traps can be applied to model current injection. These results shed light on the mechanism of charge injection and transport in polymers. Although PF2/6 has a high degree of interchain disorder, the as-is polymer shows regions of structural ordering that resembles the hexagonal phase $;^{15}$ these ordered segments are most probably responsible for charge injection and transport. The SCLC model with discrete shallow-trap levels may be applicable to other amorphous polymers which have regions of ordering. A cautionary remark is that the true SCLC behavior is observed only in the first bias sweep in the as-is polymer, an effect that may be easily overlooked, since the injected carriers at voltages higher than $V_{T F L}$ generates a new set of exponential traps.

\section{ACKNOWLEDGMENT}

We gratefully acknowledge the support of this work through the National Science Foundation under Grant Nos. ECS-0523656 and DMR-0413601.
*Corresponding author. Electronic address: guhas@missouri.edu

${ }^{1}$ M. Leclerc, J. Polym. Sci., Part A: Polym. Chem. 39, 2867 (2001).

${ }^{2}$ U. Scherf and E. J. W. List, Adv. Mater. (Weinheim, Ger.) 14, 477 (2002).

${ }^{3}$ A. L. Burin and M. A. Ratner, J. Polym. Sci., Part B: Polym. Phys. 41, 2601 (2003).

${ }^{4}$ L.-L. Chua, J. Zaumseil, J.-F. Chang, E. C.-W. Ou, P. K.-H. Ho, H. Sirringhaus, and R. H. Friend, Nature (London) 434, 194 (2005).

${ }^{5}$ P. E. Parris, V. M. Kenkre, and D. H. Dunlap, Phys. Rev. Lett. 87, 126601 (2001).

${ }^{6}$ M. N. Bussac and L. Zuppiroli, Phys. Rev. B 55, 15587 (1997).

${ }^{7}$ R. Schmechel and H. von Seggern, Phys. Status Solidi A 201, 1215 (2004).

${ }^{8}$ W. Graupner, G. Leditzky, G. Leising, and U. Scherf, Phys. Rev. B 54, 7610 (1996).

${ }^{9}$ P. D. Townsend and R. H. Friend, Phys. Rev. B 40, 3112 (1989).

${ }^{10}$ A. J. Campbell, D. D. C. Bradley, and D. G. Lidzey, J. Appl. Phys. 82, 6326 (1997).

${ }^{11}$ M. A. Lampert and P. Mark, Current Injection in Solids (Academic, New York, 1970).

${ }^{12}$ A. Carbone, B. K. Kotowska, and D. Kotowski, Phys. Rev. Lett. 95, 236601 (2005).

${ }^{13}$ I. Musa, S. J. Higgins, and W. Eccleston, J. Appl. Phys. 81, 2288 (1997)

${ }^{14}$ M. Pope and C. E. Swenberg, Electronic Processes in Organic
Crystals and Polymer (Oxford University Press, Oxford, 1999).

${ }^{15}$ B. Tanto, S. Guha, C. M. Martin, U. Scherf, and M. J. Winokur, Macromolecules 37, 9438 (2004).

${ }^{16} \mathrm{~S}$. H. Chen, A. C. Su, C. H. Su, and S. A. Chen, Macromolecules 38, 379 (2005).

${ }^{17}$ M. Knaapila, R. Stepanyan, B. P. Lyons, M. Torkkeli, T. P. A. Hase, R. Serimaa, R. Güntner, O. H. Seeck, U. Scherf, and A. P. Monkman, Macromolecules 38, 2744 (2005).

${ }^{18}$ M. Arif, C. Volz, and S. Guha, Phys. Rev. Lett. 96, 025503 (2006).

${ }^{19}$ M. Yun, R. Ravindran, M. Hossain, S. Gangopadhyay, U. Scherf, T. Bünnagel, F. Galbrecht, M. Arif, and S. Guha, Appl. Phys. Lett. 89, 013506 (2006).

${ }^{20}$ D. Poplavskyy, W. Su, and F. So, J. Appl. Phys. 98, 014501 (2005).

${ }^{21}$ A. Kadashchuk, R. Schmechel, H. von Seggern, U. Scherf, and A. Vakhnin, J. Appl. Phys. 98, 024101 (2005).

${ }^{22}$ D. V. Lang, X. Chi, T. Siegrist, A. M. Sergent, and A. P. Ramirez, Phys. Rev. Lett. 93, 076601 (2004).

${ }^{23}$ S. Nespurek and J. Sworakowski, J. Appl. Phys. 51, 2098 (1980).

${ }^{24}$ S. Gangopadhyay, S. Iselborn, H. Rübel, B. Schröder, and J. Geiger, Philos. Mag. Lett. 51, L33 (1985).

${ }^{25}$ M. A. Rafiq, Y. Tsuchiya, H. Mizuta, S. Oda, S. Uno, Z. A. K. Durrani, and W. I. Mine, Appl. Phys. Lett. 87, 182101 (2005).

${ }^{26}$ T. Kreouzis, D. Poplavskyy, S. M. Tuladhar, M. Campoy-Quiles, J. Nelson, A. J. Campbell, and D. D. C. Bradley, Phys. Rev. B 73, 235201 (2006). 